\title{
海外招待講演
}

\section{Clinical Implications of Nasopharyngeal Bacterial Colonization}

\author{
Timothy F. Murphy \\ Division of Infectious Diseases, Department of Medicine, University at Buffalo, \\ the State University of New York, Buffalo NY USA
}

Nasopharyngeal colonization by bacteria is a dynamic and complex process. The acquisition of bacteria in the nasopharynx is the first step in the pathogenesis of several important infections including otitis media, sinusitis, pneumonia, and invasive pneumococcal disease. There is a strong relationship between the frequency of colonization by the pneumococcus, H. influenzae, and M. catarrhalis with otitis media. Widespread immunization with pneumococcal conjugate vaccine has resulted in changing patterns of colonization and may also be causing a change in the distribution of pathogens that cause otitis media. Mucosal immunization is a potentially useful approach for vaccine development for $H$. influenzae. Early clinical trials provide evidence that vaccines are effective in the prevention of otitis media. It will be important to monitor the effect of new vaccines on patterns of nasopharyngeal colonization.

\section{Introduction}

Bacterial colonization of the human nasopharynx is a critical first step in the pathogenesis of several infections of the respiratory tract. However, infection that produces clinical symptoms is actually not the most common outcome of bacterial colonization. In most cases, bacteria colonize the nasopharynx and do not cause disease. However, in a minority of cases, several important clinical diseases may result. These include otitis media, sinusitis and pneumonia. In addition, hematogenous dissemination of Streptococcus pneumoniae (pneumococcus) from the respiratory tract may occur. The three most common bacterial pathogens of the respiratory tract that result initially from nasopharyngeal colonization are S. pneumoniae, Haemophilus influenzae, in particular nontypeable or nonencapsulated strains, and Moraxella catarrhalis. Each of these bacteria has multiple adhesins on their surface that mediate attachment to the human respiratory epithelium.

\section{Patterns of Nasopharyngeal Colonization}

The rate of colonization by these important bacterial pathogens is highly dependent on age in that the rate of asymptomatic colonization decreases with age. In prospective studies pre-school age children (under age 5) show a substantially higher rate of colonization than do older children and adults. It is interesting that the age distribution of otitis media parallels this pattern of nasopharyngeal colonization, consistent with the key role that nasopharyngeal colonization in the pathogenesis of otitis media (see below).

Several patterns of colonization are observed in the first year of life. Some strains are rapidly cleared and never return. By contrast, some episodes of colonization are prolonged, and the same strain can persist for several months at a time. Finally, a common pattern is sequential colonization with different strains.

\section{Nasopharyngeal Colonization and Otitis Media}

The first step in the pathogenesis of otitis media is the acquisition of a bacterial strain into the nasopharynx. Several well-established risk factors are associated with clinical otitis media following nasopharyngeal colonization. These include exclusive formula feeding, attending day care centers, number of siblings, crowded living conditions, 
genetic background, smoking in the household, sleeping position, episodes of viral upper respiratory tract infection, and pneumococcal conjugate vaccine status.

Otitis media is a highly prevalent worldwide problem that is associated with significant morbidity. Approximately 80 per cent of all children will have an episode of otitis media by the age of 3 years . In developed countries, otitis media is the most common reason for visits to the doctor and the most common reason for which antibiotic therapy is administered. Children who are otitis prone suffer the most from otitis media because chronic and recurrent infections are associated with hearing loss and with delays in speech and language development ${ }^{1,2}$. These are the children who would benefit most from a vaccine to prevent otitis media.

In a prospective study, Faden and colleagues ${ }^{3}$ studied the relationship of nasopharyngeal colonization and the development of otitis media in children. They enrolled 306 infants at birth and performed monthly cultures and followed the children for otitis media. The frequency of episodes of otitis media showed a strong relationship with the frequency of colonization by pneumococcus, H. influenzae, and M. catarrhalis. This study and others show the important relationship between otitis media and nasopharyngeal colonization, and highlight the importance of nasopharyngeal colonization in the pathogenesis of otitis media.

\section{Effect of Pneumococcal Conjugate Vaccines on Nasopharyngeal Colonization}

The widespread use of pneumococcal conjugate vaccines in many developed countries has altered patterns of nasopharyngeal colonization substantially. This vaccine is comprised of pneumococcal polysaccharide from seven serotypes that are conjugated to a protein carrier. Universal immunization was recommended in the United States in 2000, and many other countries are immunizing a large proportion of their children. This has resulted in a large reduction in invasive pneumococcal infection in infants and children. One of the mechanisms of this effect is the reduction in nasopharyngeal colonization by the pneumococcus. For example, Millar and colleagues ${ }^{4}$ demonstrated that children who received the pneumococcal conjugate vaccine had a significantly lower rate of colonization by vaccine types compared to control children. Interestingly, they also demonstrated that the immunized children had a higher rate of colonization by non-vaccine serotypes, a phenomenon known as serotype replacement.

Several studies have assessed the potential clinical implications of alteration of nasopharyngeal colonization. Revai and colleagues ${ }^{5}$ demonstrated that the prevalence of otitis media caused by $H$. influenzae and M. catarrhalis is trending upward in the years during which children have been receiving the pneumococcal conjugate vaccine compared to years prior to the widespread use of the vaccine. In addition, Block and colleagues ${ }^{6}$ and Casey and Pichichero ${ }^{7}$ have shown similar trends. It will be important to follow the changing epidemiology of nasopharyngeal colonization as vaccines that affect nasopharyngeal colonization patterns are developed and used.

Finally, an interesting effect has been observed with widespread use of the pneumococcal conjugate vaccines. The incidence of invasive disease in adults has decreased substantially as a result of immunization of infants and children ${ }^{8}$. This so-called herd effect is a result of the reduction of nasopharyngeal colonization by vaccine serotypes in infants and children and thus reduced transmission to adults.

\section{Vaccines for Otitis Media}

Vaccines to prevent otitis media would have an enormous beneficial effect, particularly for children who are otitis prone. In view of the observation that otitis media is a mucosal infection, there has been much interest in exploiting mucosal immunization in an effort to prevent such infections. In the case of $H$. influenzae, outer membrane protein P6 has been the subject of intensive investigation as a vaccine antigen. In a series of studies, Hotomi and colleagues ${ }^{9}$ 10) have demonstrated that intranasal immunization with recombinant P6 induces a specific mucosal immune response in mice and results in enhanced clearance of nasopharyngeal colonization. In a second study, Ostberg and colleagues ${ }^{11}$ showed that mucosal immunization with outer membrane protein P2 of $H$. influenzae induces antibodies to potentially protective epitopes, whereas systemic immunization failed to do so. These studies indicate that mucosal immunization is 
a viable strategy for vaccine development for $H$. influenzae and perhaps for other pathogens that cause otitis media.

A vaccine to prevent otitis media caused by the pneumococcus and by $H$. influenzae has been developed and tested in clinical trials. This vaccine consists of pneumococcal polysaccharide from 11 serotypes conjugated to protein D of $H$. influenzae. Protein D is a conserved protein antigen on the surface of $H$. influenzae. Prymula and colleagues ${ }^{12}$ demonstrated in a randomized controlled prospective clinical trial that immunization with this vaccine induced serotype-specific protective responses against pneumococcal otitis media and for the first time showed clinical efficacy against nontypeable $H$. influenzae otitis media. This is an important result and provides a proof of principle for preventing otitis media by immunization with conjugate vaccines. It will be important to assess the effect of this vaccine on nasopharyngeal colonization and also to develop the vaccine further so that better efficacy can be obtained.

This paper was presented at the $48^{\text {th }}$ Annual Meeting of the Japan Rhinological Society in Matsue City, Shimane prefecture, Japan on October 2, 2009.

\section{References}

1) Faden $\mathrm{H}$ : The microbiologic and immunologic basis for recurrent otitis media in children. Eur J Pediatr 2001; 160 : 407-13.

2 ) Teele DW, Klein JO, Chase C, et al: Otitis media in infancy and intellectual ability, school achievement, speech, and language at age 7 years. J Infect Dis 1990 ; 162 : 685-94.

3 ) Faden H, Duffy L, Wasielewski R, et al : Relationship between nasopharyngeal colonization and the development of otitis media in children. J Infect Dis 1997 ; 175 : 1440-5.

4 ) Millar EV, O’Brien KL, Watt JP, et al : Effect of community-wide conjugate pneumococcal vaccine use in infancy on nasopharyngeal carriage through 3 years of age : a cross-sectional study in a high-risk population. Clin Infect Dis $2006 ; 43: 8-15$.

5 ) Revai K, Mamidi D, Chonmaitree T: Association of nasopharyngeal bacterial colonization during upper respiratory tract infection and the development of acute otitis media. Clin Infect Dis 2008; 46 : e34-7.

6 ) Block SL, Hedrick J, Harrison CJ, et al: Community-wide vaccination with the heptavalent pneumococcal conjugate significantly alters the microbiology of acute otitis media. Pediatr Infect Dis J 2004 ; 23 : 829-33.

7 ) Casey JR, Pichichero ME: Changes in frequency and pathogens causing acute otitis media in 1995-2003. Pediatr Infect Dis J 2004 ; $23: 824-8$.

8 ) Lexau CA, Lynfield R, Danila R, et al: Changing epidemiology of invasive pneumococcal disease among older adults in the era of pediatric pneumococcal conjugate vaccine. JAMA 2005 ; 294 : 2043-51.

9 ) Hotomi M, Yamanaka N, Shimada J, et al : Intranasal immunization with recombinant outer membrane protein P6 induces specific immune responses against nontypeable Haemophilus influenzae. Int J Pediatr Otorhinolaryngol $2002 ; 65$ : 109-16.

10) Hotomi M, Ikeda Y, Suzumoto M, et al : A recombinant P4 protein of Haemophilus influenzae induces specific immune responses biologically active against nasopharyngeal colonization in mice after intranasal immunization. Vaccine $2005 ; 23: 1294-300$.

11) Ostberg KL, Russell MW, Murphy TF : Mucosal immunization of mice with recombinant OMP P2 induces antibodies that bind to surface epitopes of multiple strains of nontypeable Haemophilus influenzae. Mucosal Immunol $2009 ; 2: 63-73$.

12) Prymula R, Peeters P, Chrobok V, et al: Pneumococcal capsular polysaccharides conjugated to protein D for prevention of acute otitis media caused by both Streptococcus pneumoniae and non-typable Haemophilus influenzae : a randomised double-blind efficacy study. Lancet 2006 ; 367 : 740-8. 